Preprint typeset in JHEP style - HYPER VERSION

Saclay t02/020

\title{
On Anomalies in Orbifold Theories
}

\author{
Luigi Pilo \\ Service de Physique Theórique, CEA/Saclay, F-91191 Gif-sur-Yvette, France \\ E-mail: pilo@spht.saclay.cea.fr \\ Antonio Riotto \\ Dipartimento di Fisica dell'Università di Padova and INFN Sezione di Padova \\ Via Marzolo 8, I-35131 Padova, Italy \\ E-mail: antonio.riotto@pd.infn.it
}

\begin{abstract}
We study the issue of gauge invariance in five-dimensional theories compactified on an orbifold $S^{1} /\left(\mathbb{Z}_{2} \times \mathbb{Z}_{2}^{\prime}\right)$ in the presence of an external $U(1)$ gauge field. From the fourdimensional point of view the theory contains a tower of Kaluza-Klein Dirac fermions with chiral couplings and it looks anomalous at the quantum level. We show that this "anomaly" is cancelled by a topological Chern-Simons term which is generated in the effective action when the gauge theory is regularized introducing an Pauli-Villars fermion with an odd mass term. In the presence of a classical background gauge field, the fermionic current acquires a vacuum expectation value, thus generating the suitable Chern-Simons term and a gauge invariant theory.
\end{abstract}

Keywords: Anomalies, Field Theories in Higher Dimensions, Orbifold Theories. 


\section{Introduction}

The presence of extra-dimensions is a crucial ingredient in theories explaining the unification of gravity and gauge forces. A typical example is string theory where more than three spatial dimensions are necessary for the consistency of the theory. It has recently become clear that extra-dimensions may be very large and could be even testable in accelerator experiments [1]. Of special interest are the theories on orbifold spaces [2] which are obtained compactifying the extra-dimensions and imposing a discrete symmetry acting on the higher dimensional coordinates. The four-dimensional (4D) low energy effective field theory coming from an orbifold compactification contains a tower of Kaluza-Klein (KK) states and may be chiral. The corresponding 4D orbifold gauge theory may therefore be anomalous [3] - signaling the breaking of gauge invariance - unless anomaly cancellation takes place through some non-trivial mechanism such as the Green-Schwarz [4] or the bulk inflow mechanisms [5].

The gauge anomaly in five-dimensional (5D) theories compactified on an $S^{1} / \mathbb{Z}_{2}$ orbifold with chiral boundary conditions for a single bulk fermion with unit charge under an abelian gauge group $U(1)$ was first discussed in Ref. [6]. The anomaly - defined as the five dimensional divergence of the current - lives entirely on the orbifold fixed planes

$$
\partial_{M} J^{M}=\frac{1}{2}[\delta(y)+\delta(y-\pi R)] \mathcal{Q}(x, y),
$$

where $J^{M}$ is the $5 \mathrm{D}$ fermionic current and

$$
\mathcal{Q}(x, y)=\frac{g_{5}^{2}}{32 \pi^{2}} F_{\mu \nu}(x, y) \widetilde{F}^{\mu \nu}(x, y),
$$

is the $4 \mathrm{D}$ chiral anomaly in the external gauge potential $A_{M}(x, y)^{1}$. Therefore four dimensional anomaly cancellation is sufficient to ensure the consistency of the higher dimensional orbifold theory. However, it was recently claimed that this phenomenon does not persist [7] in a five-dimensional field theory with a $U(1)$ gauge field and a charged fermion, compactified on the orbifold $S^{1} /\left(\mathbb{Z}_{2} \times \mathbb{Z}_{2}^{\prime}\right)$. Despite the fact that the orbifold projections remove both fermionic zero modes, gauge anomalies localized at the fixed points were found

$$
\partial_{M} J^{M}=\frac{1}{4}[\delta(y)-\delta(y-\pi R / 2)+\delta(y-\pi R)-\delta(y-3 \pi R / 2)] \mathcal{Q}(x, y) .
$$

The $4 \mathrm{D}$ effective theory is anomaly-free because anomalies cancel after integration over the fifth dimension, but gauge invariance is broken, spoiling the consistency of the 5D theory. This result would be important for phenomenologically interesting models as the one discussed in Ref. [8] whose light spectrum contains just the zero modes of the Standard Model fields with an anomaly-free fermion content.

The goal of this paper is to show that gauge invariance in models with five-dimensions compactified on an orbifold $S^{1} /\left(\mathbb{Z}_{2} \times \mathbb{Z}_{2}^{\prime}\right)$ can be maintained if we admit the presence of an odd mass term for the Pauli-Villars regulator. This procedure leads to the appearance in

\footnotetext{
${ }^{1}$ In our notation: $M=[(\mu=0,1,2,3), 5]$ and $y=x^{5}$ is the fifth coordinate compactified on a circle with radius $R ; g_{5}$ is the $5 \mathrm{D}$ gauge coupling constant.
} 
the effective action of a Chern-Simons (CS) topological term whose gauge variation exactly cancels the anomalous term.

Our results for orbifolds theories are reminiscent of the well-known phenomenon present in theories with an odd number of non-compact dimensions where the parity-violating part of the vacuum current induced by the classical background gauge field implies the presence in the effective action of a CS topological invariant which is odd under parity transformations [9].

The paper is organized as follows. In section 2 we summarize the computation leading to the anomaly in the theory compactified on $S^{1} /\left(\mathbb{Z}_{2} \times \mathbb{Z}_{2}^{\prime}\right)$ and with a KK tower of $4 \mathrm{D}$ chiral fermions with opposite parities under the two $\mathbb{Z}_{2}$ 's. In section 3 we discuss the CS counterterm suitable to cancel the anomaly and in section 4 we show the CS counterterm is induced by a Pauli-Villars regulator. Finally, section 5 contains our conclusions.

\section{The $5 \mathrm{D}$ vector current}

Consider a 5D fermion (Dirac) living in $\mathbb{R}^{4} \times \mathcal{P}$ coupled with a $U(1)$ external gauge field. The compact component of the space is a circle $S^{1}$ of radius $R$ modulo some discrete group $\mathbb{Z}_{2} \times \mathbb{Z}_{2}^{\prime}$ (or $\mathbb{Z}_{2}$ ) which in general has fixed points. As a result, the resulting space in an orbifold. The action we consider is

$$
S=\int d^{5} x\left[i \bar{\Psi} \Gamma^{M} \partial_{M} \Psi-g_{5} \bar{\Psi} \Gamma^{M} \Psi A_{M}\right]
$$

where

$$
\Gamma^{\mu}=\left(\begin{array}{cc}
0 & \sigma^{\mu} \\
\bar{\sigma}^{\mu} & 0
\end{array}\right), \quad \Gamma^{4}=-i\left(\begin{array}{cc}
-1 & 0 \\
0 & 1
\end{array}\right)=-i \gamma^{5}, \quad \sigma^{\mu}=(1, \vec{\sigma}), \quad \bar{\sigma}^{\mu}=(1,-\vec{\sigma}) .
$$

The two orbifold projections act on the space-time points as

$$
\begin{array}{ll}
\mathbb{Z}_{2}: & \left(x^{\mu}, y\right) \rightarrow\left(x^{\mu}, y^{\prime}\right)=\left(x^{\mu}, 2 \pi R-y\right) \\
\mathbb{Z}_{2}^{\prime}: & \left(x^{\mu}, y\right) \rightarrow\left(x^{\mu}, y^{\prime}\right)=\left(x^{\mu}, \pi R-y\right) \quad y \in[0,2 \pi R] .
\end{array}
$$

and $y$ is identified with $y^{\prime}$. On the 5D spinor the following condition is imposed

$$
\Psi\left(x, y^{\prime}\right)=\epsilon \gamma^{5} \Psi(x, y), \quad \epsilon= \pm 1
$$

We shall denote by $\epsilon$ and $\epsilon^{\prime}$ the parity of the field. Notice that a $5 \mathrm{D}$ mass term $M$ is forbidden unless it has a non-trivial profile in the bulk with parities $(-,-)$. In [7] was claimed that the 5D current

$$
J^{M}=\bar{\Psi} \Gamma^{M} \Psi
$$

though is classically conserved, has an anomalous divergence at the quantum level. The argument is based on the result for the covariant anomaly for chiral fermions in four dimensions. Indeed one can rewrite the action (2.1) as a collection of $4 \mathrm{D}$ massive Dirac 
fermions by expanding $\Psi$ in terms of the complete set formed by the solutions of free Dirac equation in $5 \mathrm{D}$

$$
\begin{aligned}
& \Psi(x, y)=\sum_{n}\left[\psi_{n_{R}}(x) \phi_{n}^{R}(y)+\psi_{n_{L}}(x) \phi_{n}^{L}(y)\right] ; \\
& \psi_{n_{L / R}}=P_{L / R} \psi_{n} \quad ;
\end{aligned}
$$

where $\psi_{n}$ is a $4 \mathrm{D}$ Dirac fermion of mass $M_{n}$. The KK modes $\phi_{n}^{L / R}$ satisfy

$$
\begin{aligned}
& \phi_{n}^{R} M_{n}-\frac{d}{d y} \phi_{n}^{L}=0 \\
& \phi_{n}^{L} M_{n}+\frac{d}{d y} \phi_{n}^{R}=0
\end{aligned}
$$

From Eq. (2.4) we get the following transformation rules under the orbifold projections

$$
\phi^{R / L}(-y)= \pm \epsilon \phi^{R / L}(y) \quad \phi^{R / L}(\pi R-y)= \pm \epsilon^{\prime} \phi^{R / L}(y)
$$

The action (2.1) can be written, using the orthogonality of the KK modes, as

$$
\begin{aligned}
S=\int d^{4} x & {\left[\sum_{n}\left(\bar{\psi}_{n} i \gamma^{\mu} \partial_{\mu} \psi_{n}-M_{n} \bar{\psi}_{n} \psi_{n}\right)\right.} \\
& \left.-g_{5} \sum_{n, m}\left(j_{L m n}^{\mu} \mathcal{A}_{\mu m n}^{L}+j_{R m n}^{\mu} \mathcal{A}_{\mu m n}^{R}-i j_{5 m n} \mathcal{A}_{5 m n}\right)\right] ;
\end{aligned}
$$

where

$$
\begin{aligned}
& \mathcal{A}_{\mu}^{L / R}{ }_{m n}=\int_{0}^{2 \pi R} d y \phi_{n}^{L / R}(y) \phi_{m}^{L / R}(y) A_{\mu}(x, y) \\
& \mathcal{A}_{5 m n}=\int_{0}^{2 \pi R} d y \phi_{m}^{L}(y) \phi_{n}^{R}(y) A_{5}(x, y) ;
\end{aligned}
$$

and

$$
\begin{aligned}
& j_{L / R}^{\mu} m n=\bar{\psi}_{m} \gamma^{\mu} P_{L / R} \psi_{n} \quad ; \\
& j_{5 m n}=\bar{\psi}_{L} m \psi_{R} n-\bar{\psi}_{R} n \psi_{L} m
\end{aligned}
$$

Let us now reproduce the results in [6] and [7]. After setting $A_{5}=0$ by a gauge choice ${ }^{2}$, from the classical equations of motion and the well known result for the anomalous divergence of chiral current in $4 \mathrm{D}$ one has

$$
\begin{gathered}
\partial_{\mu} j_{L m n}^{\mu}=i\left[\bar{\psi}_{m} M_{m} P_{L} \psi_{n}-\bar{\psi}_{m} P_{R} M_{n} \psi_{n}\right]-\frac{g_{5}^{2}}{32 \pi^{2}}\left(\mathcal{F}_{\mu \nu}^{L}{\widetilde{\mathcal{F}_{L}}}^{\mu \nu}\right)_{m n} \\
\left.\partial_{\mu} j_{R}^{\mu}=i\left[\bar{\psi}_{m} M_{m} P_{R} \psi_{n}-\bar{\psi}_{m} P_{L} M_{n} \psi\right)_{n}\right]+\frac{g_{5}^{2}}{32 \pi^{2}}\left(\mathcal{F}_{\mu \nu}^{R} \widetilde{\mathcal{F} R}^{\mu \nu}\right)_{m n} .
\end{gathered}
$$

\footnotetext{
${ }^{2}$ Strictly speaking this is not allowed: one is using gauge invariance before showing that it is still a good symmetry at the quantum level.
} 
On the other hand, the 5D current can written in terms of the 4D currents

$$
\begin{aligned}
& J^{\mu}(x, y)=\sum_{m n}\left[\phi_{m}^{R}(y) \phi_{n}^{R}(y) j_{R}^{\mu}(x)+\phi_{m}^{L}(y) \phi_{n}^{L}(y) j_{L m n}^{\mu}(x)\right] \\
& J^{5}=-i \sum_{m n} \phi_{m}^{L}(y) \phi_{n}^{R}(y) j_{5 m n}(x)
\end{aligned}
$$

The fifth-dimensional structure of the divergences is recovered noticing that at the classical level

$$
\partial_{y} J^{5}=\sum_{m n} i\left[M_{n}\left(\phi_{m}^{R} \phi_{n}^{R}-\phi_{m}^{L} \phi_{n}^{L}\right) \bar{\psi}_{m}\left(P_{L}-P_{R}\right) \psi_{n}\right] .
$$

Combining Eqs.(2.13), (2.14), (2.15) and (2.16), one finally gets

$$
\partial_{M} J^{M}=\frac{g_{5}^{2}}{32 \pi^{2}} f(y) F_{\mu \nu} \widetilde{F}^{\mu \nu}, \quad f(y)=\sum_{m}\left(\phi_{m}^{R} \phi_{m}^{R}-\phi_{m}^{L} \phi_{m}^{L}\right)
$$

It should be stressed that in deriving (2.17) one is tacitly supposing that (2.16) is still valid at the quantum level and all the quantum effects are encoded in Eqs. (2.13-2.14); we will show that actually this is not the case.

The sum over the KK modes in (2.17) can be computed and it reads in the case $\mathcal{P}=\mathbb{Z}_{2} \times \mathbb{Z}_{2}^{\prime}$ for the various choices of parity for the fermion

$$
\begin{aligned}
& f^{(++)}(y)=-f^{(--)}(y)=\frac{1}{4 R} \sum_{n=-\infty}^{+\infty} \delta(y / R-n \pi / 2) ; \\
& f^{(+-)}(y)=-f^{(-+)}(y)=\frac{1}{4 R} \sum_{n=-\infty}^{+\infty}(-1)^{n} \delta(y / R-n \pi / 2)
\end{aligned}
$$

In particular, if the fermions have opposite parities $(+,-)$ and $(-,+)$, one recovers Eq. (1.3). For the case $\mathcal{P}=\mathbb{Z}_{2}$ one finds

$$
f^{(+)}(y)=-f^{(-)}(y)=\frac{1}{2 R} \sum_{n=-\infty}^{+\infty} \delta(y / R-\pi n),
$$

which reproduces Eq. (1.1). ${ }^{3}$

\section{Deformed Chern-Simons counterterm}

In general, the manifestation of an anomaly at the quantum level reflects the failure of removing the ultraviolet divergences and - at the same time - preserving all the classical symmetries of the theory.

The natural question is therefore whether there exists a local counterterm $S_{\mathrm{ct}}$ such that the new action $S^{\prime}=S_{5 D}+S_{\text {ct }}$ leads to a conserved $5 \mathrm{D}$ vector current in the orbifold theory $S^{1} /\left(\mathbb{Z}_{2} \times \mathbb{Z}_{2}^{\prime}\right)$ maintaining the symmetries of classical action.

\footnotetext{
${ }^{3}$ Eqs. (2.18)-(2.20) still hold when a bulk mass term $M(y)$ for the $5 \mathrm{D}$ fermion is present. Indeed, one can compute $f(y)$ simply by using the completeness of the KK modes without relying on their explicit form.
} 
Consider the vacuum functional $Z[A]$

$$
Z[A]=e^{i W[A]}=\int D[\Psi] e^{i S[A, \Psi]} ;
$$

a would-be anomaly $G$ shows up in a non-zero variation of the connected generating functional $W$ under a gauge transformation $\delta A_{M}=-\partial_{M} \lambda$ of the the vector potential

$$
\delta_{\lambda} W[A]=i \int d^{5} x \lambda G[A]
$$

As a result, one has for the divergence of the current

$$
\partial_{M}\left\langle J^{M}\right\rangle_{\text {conn. }}=G[A], \quad \frac{\delta W[A]}{\delta A_{M}}=\left\langle J^{M}\right\rangle_{\text {conn. }} .
$$

$G$ is defined modulo a local functional of $A$, and the presence of a true anomaly is related to the impossibility of finding a suitable $S_{\text {ct }}$ such that $G$ is zero. The natural candidate for $S_{\text {ct }}$ in our case is the following deformed Chern-Simons term

$$
S_{\mathrm{CS}}=\int u(y) A \wedge F \wedge F=\int d^{5} x \epsilon^{M N O P Q} A_{M} F_{N O} F_{P Q} u(y) .
$$

The counterterm $S_{\mathrm{CS}}$ will be consistent with the orbifold projections if $u$ has parity $(-,-)$. The gauge variation of the new connected generating functional is given by

$$
\delta_{\lambda} W^{\prime}=i \int d^{5} x \lambda\left[G[A]+F_{\mu \nu} \widetilde{F}^{\mu \nu} \partial_{y} u(y)\right],
$$

and the condition of vanishing divergence for the current gives the following constraint on the function $u(y)$

$$
\partial_{y} u(y)+\frac{g_{5}^{2}}{32 \pi^{2}} f(y)=0
$$

In the case $(+,-)$ and $(+,-)$ a solution is easily found

$$
u^{(+-)}(y)=-u^{(-+)}(y)=-\frac{g_{5}^{2}}{128 \pi^{2}} \frac{1}{2} \operatorname{sgn}_{\pi}(\pi / 2-y / R)
$$

where $\operatorname{sgn}_{\pi}(x)$ is the sign function periodically extended with period $\pi$. The other cases are more subtle; if we suppose that $u(y)=u(y+2 \pi R)$ then Eq. (3.6) can be solved only if

$$
\int_{0}^{2 \pi R} f(y) d y=0
$$

As a result, there is no periodic $u$ which solves Eq. 3.6) in the cases of fermions with parities $(+,+)(-,-)$ where a chiral zero mode is present in the spectrum, the same result holds for the case of a single $\mathbb{Z}_{2}$.

Since the CS term (3.4) one needs to add in order to preserve the gauge invariance of the theory depends on the odd function $u(y)$, it becomes clear that gauge invariance can be maintained if one accepts the presence of odd operators which requires piece-wise constant (odd) functions as generalized couplings in order to be consistent with orbifold 
symmetries. An odd operator appears naturally when the theory is regulated in a gauge invariant way as a topological term in fifth component of the the ground-state current $\left\langle J^{M}\right\rangle$. The fact that an odd operator can have a VEV is not is not in contradiction with the invariance of the vacuum under the orbifold projections, indeed, due the breaking of translation invariance invariance one can only deduce that the VEV is an odd function of $y$. As we shall demonstrate in the next section, the CS term term can be generated naturally with the proper coefficient and sign adopting a gauge invariant regularization.

The possibility of adding to the Lagrangian a CS term with an odd function $u(y)$ and a suitable coefficient was first mentioned in Ref. [7], where the odd function needed in the CS term was understood as the expectation value of a new odd field $\chi(y)$ added to the original theory to cancel the anomaly. However our point of view is different: the theory with a single 5D Dirac fermion is by itself non-anomalous, and there is no need to introduce an ad-hoc extra field.

\section{The vacuum current}

We now derive an explicit expression for the induced vacuum current in the orbifold theory $S^{1} /\left(\mathbb{Z}_{2} \times \mathbb{Z}_{2}^{\prime}\right)$ for a $5 \mathrm{D}$ fermion with parity (+-) and (-+) and for simplicity with vanishing bulk mass. We consider a gauge field background which is uniform in the bulk. The generic result can then be derived by general covariance arguments.

From the form of the CS term, one expects that the vacuum expectation value of the vector current acquires a non-vanishing component along the fifth direction

$$
\left\langle J^{5}(x, y)\right\rangle=-\frac{i}{Z[A]} \frac{\delta Z[A]}{\delta A_{5}(x, y)} .
$$

From this term one can then deduce the CS term in the effective action. We first introduce a new fermion with five-dimensional mass term $M(y) \bar{\Psi} \Psi$ which at the end of the computation we decouple from the theory by taking the mass to infinity (Pauli-Villars regularization).

Since the fermionic bilinear $\bar{\Psi} \Psi$ is odd under both the $\mathbb{Z}_{2}$ and the $\mathbb{Z}_{2}^{\prime}$ parities, the function $M(y)$ has to be odd under both reflection symmetries. For instance, we can choose $M(y)=$ $\kappa \operatorname{Sgn}_{\pi}(\pi / 2-y / R), \quad \kappa>0$, where $[\kappa]=1$. Eventually we will let $\kappa \rightarrow \infty$. With a mass term the equations for the KK modes read

$$
\begin{aligned}
& \phi_{n}^{R} M_{n}-M(y) \phi_{n}^{L}-\frac{d}{d y} \phi_{n}^{L}=0 ; \\
& \phi_{n}^{L} M_{n}-M(y) \phi_{n}^{R}+\frac{d}{d y} \phi_{n}^{R}=0 \quad .
\end{aligned}
$$

In the limit of large $\kappa$ one can infer from Eqs. (4.2) and (4.3) that the mass eigenvalues $M_{n}$ satisfy the relation

$$
\tan \left(\frac{\pi}{2} \alpha_{n} R\right)=-\frac{\alpha_{n}}{\kappa}
$$

where

$$
M_{n}^{2}=\alpha_{n}^{2}+\kappa^{2} .
$$




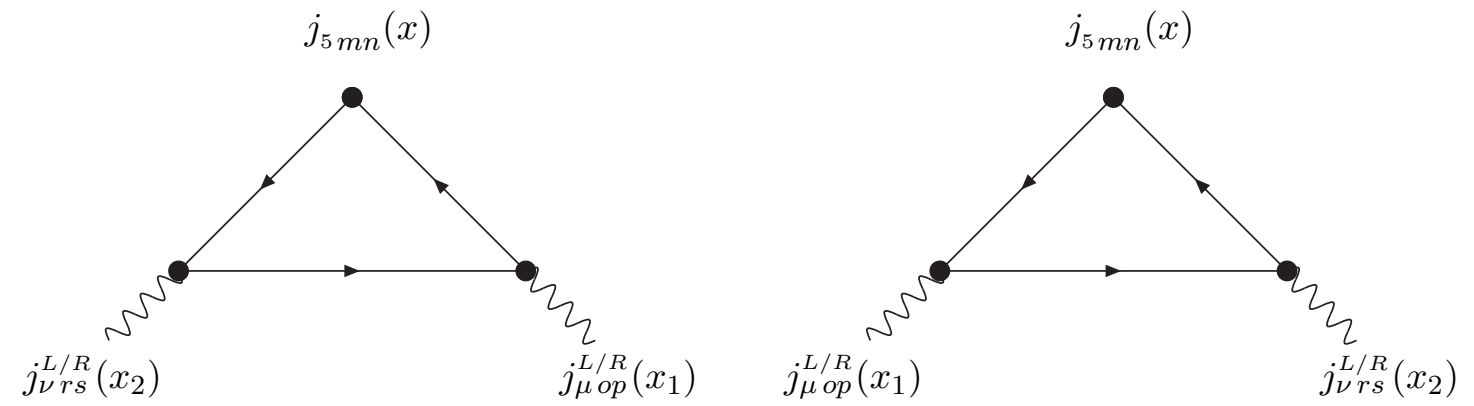

Figure 1: Triangle diagrams contributing to the vacuum expectation value of $J^{5}$

The mass eigenvalues reduce to $M_{n}^{2}=\left(\frac{2 n}{R}\right)^{2}+\kappa^{2}$ and the corresponding eigenfunctions are given by

$$
\begin{aligned}
\phi_{n}^{L} & =\frac{1}{\sqrt{\pi R}} \sin \left[\frac{2 n}{R} y\right], \\
\phi_{n}^{R} & =\frac{1}{M_{n} \sqrt{\pi R}}\left\{M(y) \sin \left[\frac{2 n}{R} y\right]+\frac{2 n}{R} \cos \left[\frac{2 n}{R} y\right]\right\} .
\end{aligned}
$$

The crucial point is that the eigenfunction $\phi_{n}^{R}$ picks up a piece which depends upon the odd mass term $M(y)$.

Our goal is to show that the decoupling procedure leaves a finite CS term in the regularized action $S_{\text {reg }}=S_{5 D}[A]-S_{5 D}[A, M]$ when the five-dimensional mass is taken to infinity.

Consider the vacuum expectation value of $J^{5}$ in the external gauge field

$$
\left\langle J^{5}(x, y)\right\rangle=-i \sum_{m n} \phi_{m}^{L}(y) \phi_{n}^{R}(y)\left\langle j_{5 m n}(x)\right\rangle .
$$

The relevant contribution to the expectation value of $j_{5}$ is the one-loop diagram in Fig. 1 where the fermions $\psi_{n_{L / R}}$ run in the loop. Selecting the part which will give the finite piece in the large $\kappa$ limit, one has

$$
\begin{array}{r}
\left\langle j_{5 m n}(x)\right\rangle=-\delta_{m n} \frac{g_{5}^{2}}{2} \int \frac{d^{4} Q_{1}}{(2 \pi)^{4}} \frac{d^{4} Q_{2}}{(2 \pi)^{4}} \frac{d^{4} q}{(2 \pi)^{4}} e^{-i x \cdot\left(Q_{1}+Q_{2}\right)} A_{\mu}(x) A_{\nu}(x) \\
\frac{8 i \epsilon^{\mu \alpha \nu \beta} Q_{1 \alpha} Q_{2 \beta} M_{n}}{\left[\left(Q_{1}+q\right)^{2}-M_{n}^{2}+i \epsilon\right]\left(q^{2}-M_{n}^{2}+i \epsilon\right)\left[\left(q-Q_{2}\right)^{2}-M_{n}^{2}+i \epsilon\right]}
\end{array}
$$

The fact that the eigenfunction $\phi_{n}^{R}$ has a piece proportional to $M(y)$ gives rise to a finite non-vanishing contribution left-over in the large $\kappa$ limit. Indeed, in this limit, the external momenta in the denominator of (4.8) can be set to zero, and we get

$$
\left\langle J^{5}(x, y)\right\rangle=-\frac{g_{5}^{2}}{32 \pi^{2}} F_{\mu \nu} \widetilde{F}^{\mu \nu} \sum_{n} \frac{1}{M_{n}} \phi_{n}^{L}(y) \phi_{n}^{R}(y) \text {. }
$$


Using the approximate form (4.6) for the modes and the relevant part in the large $\kappa$ limit, we have

$$
\left\langle J^{5}(x, y)\right\rangle=-\frac{g_{5}^{2}}{32 \pi^{2}} F_{\mu \nu} \widetilde{F}^{\mu \nu} M(y) \frac{\operatorname{Tanh}(\kappa \pi R / 2)}{8 \kappa} .
$$

The ratio $M(y) / \kappa$ does not depend upon $\kappa$ and changes sign around the fixed points. We can safely take the limit $\kappa \rightarrow \infty$ and find

$$
\left\langle J^{5}(x, y)\right\rangle=-\frac{g_{5}^{2}}{32 \pi^{2}} \operatorname{Sgn}_{\pi}(\pi / 2-y / R) \frac{1}{8} F_{\mu \nu} \widetilde{F}^{\mu \nu}
$$

Thus, the physical ground-state gets a VEV and we discover that a finite CS counterterm is produced

$$
\mathcal{L}_{\mathrm{CS}}=-\frac{g_{5}^{2}}{128 \pi^{2}} \frac{1}{2} \operatorname{Sgn}_{\pi}(\pi / 2-y / R) A_{5}(x, y) F_{\mu \nu}(x, y) \widetilde{F}^{\mu \nu}(x, y)
$$

which is the precisely the form needed in the expression (3.4) to cancel the anomaly. As a result, the cancellation of the divergence in Eq. (2.17) may be thought as coming from $\left\langle\partial_{y} J^{5}\right\rangle$, which can be non-vanishing due the lack of translation invariance in the fifth dimension. Consequently, $\left\langle\partial_{y} J^{5}\right\rangle$ gets an extra contribution because of the nontrivial shape of the function $\partial_{y} M(y)$.

Let us close with some comments about theories with a single $\mathbb{Z}_{2}$. The reader might expect that the relation between spontaneous breaking of the reflection symmetry and gauge symmetry leading to the cancellation of the anomaly described in this paper might work in that case too. However, this is not the case. The basic difference is that in 5D theories compactified on $S^{1} / \mathbb{Z}_{2}$ the odd function that one should introduce in the CS term to reproduce the singular structure in Eq. (1.1) is monotone in the bulk space with jumps every $\pi R$. The problem is that such a function is not periodic. Notice also that, with a single orbifold projection, when we take the limit $\kappa \rightarrow \infty$, the fermion does not decouple due to the chiral zero mode whose bulk wave-function becomes peaked at one of the fixed points.

\section{Conclusions}

In this paper we have studied the issue of gauge invariance in models with five-dimensions compactified on an orbifold $S^{1} /\left(\mathbb{Z}_{2} \times \mathbb{Z}_{2}^{\prime}\right)$. If, upon reduction to four-dimensions, the theory contains a tower of KK chiral fermions with opposite parities under the two $\mathbb{Z}_{2}$ 's, the theory looks anomalous at the quantum level. This would be anomaly can be canceled by a suitable topological CS term which is made even under the two reflection symmetries by the presence of an odd piece-wise constant function which is allowed by the orbifold symmetries. We have shown that such a CS term is generated if the gauge theory is regularized in a gauge invariant way This occurs because the fermionic current acquires a vacuum expectation value induced by the classical background gauge field. This phenomenon is analogous to what happens in theories with an odd number of non-compact dimensions where the parityviolating part of the vacuum current induced by a classical background gauge field implies 
the presence in the effective action of a CS topological invariant which is odd under parity transformations.

\section{Acknowledgments}

We thank F. Zwirner for useful discussions and especially R. Barbieri, R. Contino and R. Rattazzi for letting us know about their work in progress on similar issues. Work supported in part by the RTN European Program HPRN-CT-2000-00148

\section{References}

[1] V. A. Rubakov, Phys. Usp. 44, 871 (2001) [Usp. Fiz. Nauk 171, 913 (2001)] [arXiv:hep-ph/0104152].

[2] L. J. Dixon, J. A. Harvey, C. Vafa and E. Witten, Nucl. Phys. B 261, 678 (1985).

[3] S. L. Adler, Phys. Rev. 177 (1969) 2426; J. S. Bell and R. Jackiw, Nuovo Cim. A 60, 47 (1969).

[4] M. B. Green and J. H. Schwarz, Phys. Lett. B 149, 117 (1984).

[5] C. G. Callan and J. A. Harvey, Nucl. Phys. B 250, 427 (1985).

[6] N. Arkani-Hamed, A. G. Cohen and H. Georgi, Phys. Lett. B 516, 395 (2001) [arXiv:hep-th/0103135].

[7] C. A. Scrucca, M. Serone, L. Silvestrini and F. Zwirner, Phys. Lett. B 525, 169 (2002) [arXiv:hep-th/0110073].

[8] R. Barbieri, L. J. Hall and Y. Nomura, Phys. Rev. D 63, 105007 (2001) [arXiv:hep-ph/0011311].

[9] A. J. Niemi and G. W. Semenoff, Phys. Rev. Lett. 51, 2077 (1983); A. N. Redlich, Phys. Rev. Lett. 52 (1984) 18; A. N. Redlich, Phys. Rev. D 29, 2366 (1984); M. Reuter and W. Dittrich, Phys. Rev. D 33, 601 (1986). 